\title{
Theoretical Studies of AQP4 in Water \& Gas Phases, Nano Simulation of the Monte Carlo Method by Molecular Mechanics Force Fields
}

\author{
EBRAHIM SHAHMANSOORIAN ${ }^{1}$, MARYAM HASHEMY ${ }^{1}$, SAHARNAZ AHMADI ${ }^{1}$, \\ ZOHREH JAMALI ${ }^{2}$, NASTARAN ASGHARI MOGHADDAM ${ }^{1}$ and REZA RASOOLZADEH ${ }^{1,3 *}$
}

1Department of Biology, Science and Research Branch, Islamic Azad University, Tehran, Iran. 2Department of Chemistry, Science and Research Branch, Islamic Azad University, Tehran, Iran.

${ }^{3}$ Young Researchers and Elites Club, Science and Research Branch, Islamic Azad University, Tehran, Iran.

${ }^{\star}$ Corresponding author E-mail: Reza.Rasoolzadeh@yahoo.com

http://dx.doi.org/10.13005/ojc/300347

(Received: June 06, 2014; Accepted: July 23, 2014)

\begin{abstract}
Aquaporins are membrane water channels that play critical roles in controlling the water contents of cells. These channels are widely distributed in all kingdoms of life, including bacteria, plants, and mammals. More than ten different aquaporins have been found in human body, and several diseases, such as congenital cataracts and nephrogenic diabetes insipidus, are connected to the impaired function of these channels. They form tetramers in the cell membrane, and facilitate the transport of water and, in some cases, other small solutes across the membrane. However, the water pores are completely impermeable to charged species, such as protons, a remarkable property that is critical for the conservation of membrane's electrochemical potential, but paradoxical at the same time, since protons can usually be transferred readily through water molecules. The present investigation is profound manifesting of thermodynamics characteristics of the impressive AQP4 role in biology. To clarify the majestic AQP4 role in biology, it was modeled. So we use pc-based modeling and simulation software package called HyperChem by applying four different force fields. The results of our simulations have now provided new insight into the optimal stability of AQP4.
\end{abstract}

Key words: AQP4, Aquaporins, Monte Carlo, Water \& Gas Phases, Molecular Mechanics.

\section{INTRODUCTION}

Three species of all kinds of membrane proteins have been demonstrated to possess water channels properties; the aquaporins, the cotransporters, and the uniports. Water traverses from cell membrane lipid bilayer by two passage ways: One is diffusion across lipid bilayer and another is water channel reputed as aquaporin (1 and 2 3). Aquaporins are an ilk of small transmembrane proteins that predominantly facilitate water transportation across plasma 
membranes through aqueous pores that are selective, indispensable, and vital in maintaining of life ${ }^{4}$.

Peter Agre detected the first aquaporin in 1992 in red blood cells and was awarded the 2003 Nobel chemistry Prize ${ }^{5}$. Since then, 13 genres of aquaporin have been detected in animals and humans, 33 types in plants and thousand kinds in every cell membrane. We know direction of water flow is contingent on the osmotic pressure ${ }^{6}$. The water departs in a direction from a low toward high concentration of salt and nutritional substances, but the water conduits aren't always open. The Lund scientists have found out how they open and close. This was done in cooperation with a team at Chalmers University of Technology in Gothenburg, Sweden, under the supervision of Richard Neuter, and with Emad Tajkhorshid at the University of Illinois ${ }^{14}$.

Some aquaporin members pertained to aquaglyceroporins family, which transfers glycerol and other uncharged solutes as well as water. The aquaglyceroporins can be discerned from aquaporins based on amino acid sequence alignments. AQP3, the first mammalian aquaglyceroporin was cloned, is permeable to glycerol and water ${ }^{10}$.

As well as AQP7 and AQP10 convey water, glycerol, and urea when expressed in Xenopus oocytes. Moreover AQP9 transfers water, glycerol, and urea, but it also is permeable to a vast range of other solutes in oocytes ${ }^{13}$. In addition to this mammalian 13- family members, there are many alternative cell types well known as classic AQP that are just selective to water, for instance: AQP1, AQP2, AQP4, AQP5 and AQPZ related to E.Coli that is identified by X-ray Crystallography techniques ${ }^{15}$.

AQP4 is a protein of aquaporin's 13- family members that is denominated from AQP0- AQP12 .It was originally cloned in rats's brains and lungs as a plenty of new aquaporin were expressed in the brain by homology cloning.After expressing in venous oocytes, it indicates mercurial-insensitive osmotic water permeability and named the" mercurial-insensitive water channel (MIWC)". AQP4 is expansively disseminated in various organs in human body such as kidneys, eyes and the brain. Malfunctions of aquaporins are associated with diseases such as nephrogenic diabetes insipidus and Sj"ogren's syndrome ${ }^{20}$. Aquaporin water and solute permeability has been further implicated in lung and brain edema, obesity, tumor angiogenesis, and wound healing, to name just a few. Many studies have been fulfilled in the structure and function of aquaporins. Moreover, sequence analysis of the aquaporins family manifests highly homology among human series (HS) members.

Only three aquaporins have been explicitly recognized in human brain cells in vivo: $A Q P 1, A Q P 4$ and AQP9 .Veritably, AQP4 is further abundantly expressed in the brain than AQP1 or AQP9. It is cardinal and prevailing water channel in the central nervous system, where it has been enunciated to be involved in dynamic regulation, water homeostasis and pathophysiological roles (2 and 3). The significant role of AQP4 is to administer water flow in the brain. The AQP4 gene encodes two discrete mRNA with different translation initiating methionines, M1 and M23, and two corresponding forms of AQP4 proteins are synthesized. Human AQP4 was also replicated from the fetal brain cDNA library.

It is studied many simulations on AQP4. One of them is accomplish on the mechanism of water passage through AQP4 of rat brain cells via molecular dynamics simulation.In other likeness investigation, AQP4 was embedded into a bilayer member of oleylephosphatidyl choline and water. Results showed that tetramer quaternary structure of pore, organized hydrophobic amino acids residues and obtained the Brownian dynamics of free energy for water transmission via AQP4 and gave the general scheme of channel. In other research, $\mathrm{Pf}$, channel osmotic permeability coefficient was measured (7 and 12).

In another work, The AQP4 was embedded into a bilayer made of dimystroilphosPhenyl choline (DMPC).This simulation divulged a bipolar orientation ordering of the channel water that matches the pseudo symmetric aquaporins architecture from both ends of the channel.The results depict that water molecules stir through AQP4's channel by changing orientation of oxygen of each water molecule. 
Authors are encouraged to have their contribution checked for grammar. Abbreviations are allowed but should be spelt out in full when first used. Integers ten and below are to be spelt out. Italicize foreign language phrases (e.g. Latin, French).

The text is to be typeset in $10 \mathrm{pt}$ roman, single spaced with baselineskip of $13 \mathrm{pt}$. Text area is 5 inches in width and the height is 8 inches (including running head). Final pagination and insertion of running titles will be done by the publisher. Upon acceptance, authors are required to submit their data source file including postscript files for figures.

It is considerable that in recent years, computer simulations are rapidly pioneered as a scientific tool and become indispensable in many numbers of scientific fields such as biology. This process includes choosing a model; finding a way of implementation that models in a form that can be run on a computer; calculating the output of the algorithm; visualizing and studying the resultant data ${ }^{9}$. They have profound implications for our understanding the structure of theories, energy, stability, thermodynamic properties, etc. Owing to worthwhile and momentous role of AQP4 in life, it was modeled (8 and 11). Hereupon the purpose of this paper is to provide the optimal stability comprehension of AQP4 that can be advantageous in pathophysiological action ${ }^{21}$.

\section{Computational Procedure}

The protein Data Bank (PDB) is a repository for the 3D structural data of large biological molecules. At first, we get three dimensional AQP4 structure from PDB, and afterward it is opened by modeling and simulation software called "HyperChem". Simulation is performed by exerting the Single point, Geometry optimization, Molecular dynamics, Langevin dynamics and Monte carlo computation procedures.

We can perceive the most stability and minimized energy of the molecule via using of four force fields: OPLS, AMBER, CHARMM, and MM+ (16 and 19).

Finally, trajectory successive tracks are run for computation of Total energy ( $E$ tot), Gradient, Bond, Angle, Dihedral, Vander Waals (V $\mathrm{dw}$ ) and Electrostatic energy (Es) in water and gas phases $^{17-18}$.

\section{RESULTS AND DISCUSSION}

Numerous key discoveries in biology have been appeared from studies of AQP4. In current inquiry we use $\mathrm{QM} / \mathrm{MM}$ (quantum mechanics/molecular mechanics) hybrid methods. The combined QM/MM approach is a molecular simulation method that cooperates with both QM (accuracy) and MM (speed) calculations, thus allowing to study of chemical processes in solution and in proteins. The $\mathrm{QM} / \mathrm{MM}$ approach was represented in 1976 paper of Warshel and Levitt. This method enables the modeling of reactive bimolecular systems at a reasonable computational endeavor while providing the intransitive precision. Quantum mechanics method is generally applicable and allows the calculation of ground and excited state properties such as; atomic charges. Molecular mechanics describe the energy cost from ideal geometry and minimizing via force fields ${ }^{22}$. The major goal is to explore the most stability and the stable conformation of the molecule. In the QM/ MM frame, electrostatic interplays among the QM and MM QAP4 atoms were calculated by exerting four force fields. Therefore, force fields are used to calculate the energy and geometry of a molecule. It is a collection of atom types (to define the atoms in a molecule), parameters (for bond lengths, bond angles, etc.) and equations (to calculate the energy of a molecule).

Eventually, AMBER, CHARMM, OPLS and $\mathrm{MM}+$ are four unique and considerable force fields which were applied. AMBER is widely used for proteins and DNA.CHARMM is vastly used for both small molecules and macromolecules. It assigns the lowest energy.MM+ is deals with vibration motion of atoms and OPLS is optimized potential for liquid simulations. Hereupon to clarify the efficacy of AQP4 energy on QM/MM computation, the most usual explanation for total potential energy is rendered by the following equation:

The two alternative calculated energy quantities are kinetic and total energy values. Kinetic energy is an exquisite yardstick for estimating the rate of AQP4 movement as long as simulation foregoes. In symbol: 

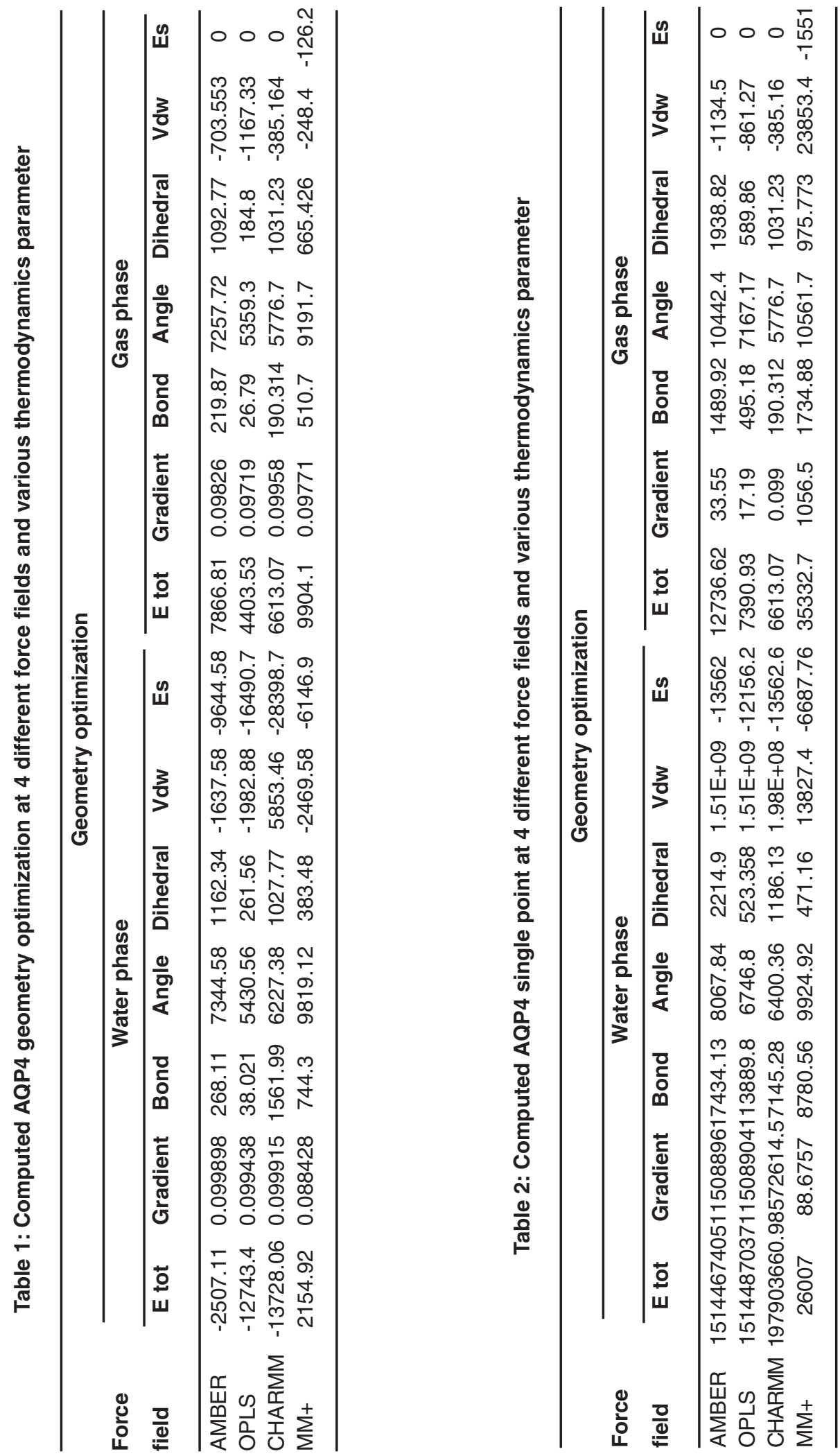
Table 3: Computed AQP4 Kinetic energy at different methods and force fields

\begin{tabular}{|c|c|c|}
\hline $\begin{array}{l}\text { Molecular dynamics } \\
\text { Kinetic energy (kcal/mol) }\end{array}$ & Gas phase & Water phase \\
\hline & $\begin{array}{l}\text { Charmm force field } \\
2820\end{array}$ & $\begin{array}{l}\text { Amber force field } \\
2814.5\end{array}$ \\
\hline \multicolumn{3}{|l|}{ Langevin dynamics } \\
\hline \multirow[t]{3}{*}{ Kinetic energy (kcal/mol) } & Gas phase & Water phase \\
\hline & $\mathrm{MM}^{+}$force field & $\mathrm{MM}^{+}$force field \\
\hline & 2410 & 2152.88 \\
\hline \multicolumn{3}{|c|}{$\begin{array}{l}\text { Table 4: Computed AQP4 Potential energy } \\
\text { at different methods and force fields }\end{array}$} \\
\hline \multicolumn{3}{|l|}{ Langevin dynamics } \\
\hline \multirow[t]{2}{*}{ Potential energy (kcal/mol) } & Gas phase & Water phase \\
\hline & $\begin{array}{l}\text { MM+ force field } \\
14729\end{array}$ & $\begin{array}{l}\text { MM+ force field } \\
12555.7\end{array}$ \\
\hline \multicolumn{3}{|l|}{ Monte carlo } \\
\hline \multirow[t]{3}{*}{ Potential energy (kcal/mol) } & Gas phase & Water phase \\
\hline & Opls force field & Amber force field \\
\hline & 6263.67 & -1160.06 \\
\hline \multicolumn{3}{|c|}{$\begin{array}{l}\text { Table 5: Computed AQP4 Total energy } \\
\text { at different methods and force fields }\end{array}$} \\
\hline \multicolumn{3}{|l|}{ Molecular dynamics } \\
\hline \multirow[t]{2}{*}{ Total energy (kcal/mol) } & Gas phase & Water phase \\
\hline & $\begin{array}{l}\text { Charmm force field } \\
12243\end{array}$ & $\begin{array}{l}\text { Amber force field } \\
13348.2\end{array}$ \\
\hline \multicolumn{3}{|l|}{ Langevin dynamics } \\
\hline \multirow[t]{3}{*}{ Total energy (kcal/mol) } & Gas phase & Water phase \\
\hline & $\mathrm{MM}+$ force field & $\mathrm{MM}+$ force field \\
\hline & 12488.3 & 14731.1 \\
\hline
\end{tabular}

$K=1 / 2\left(\Sigma m v^{2}\right)=3 / 2 K T$

For assay the optimum QAP4 energy, the total energy is presented by sum of kinetic and potential energy that is recognized as HAMILTONIAN energy. In symbols the total energy

$$
\mathrm{E}_{\text {Total }}=\Sigma \mathrm{E}_{\text {Potential }}+\Sigma \mathrm{E}_{\text {Kinetic }}
$$

As recitation before, $\mathrm{AQP} 4$ was treated at QM/MM trajectory. To elevate the analysis and to explain the results of calculations all computational data at two phases, diverse force fields and calculation methods were compiled and compared with each other.From a statistical point of view, geometry optimization represents optimum structure molecule state and the most stability of system energy, macromolecule, etc.

Therefore, according to results of Table 1 , based on the related data, the most total energy stables are $4403.53 \mathrm{Kcal} / \mathrm{mol}$ and $-13728.06 \mathrm{Kcal} /$ 
mol value at gas and water phase respectively. Accordingly the most stability was seen at water phase at CHARMM force field. Alike in obtain receive of data, angle and dihedral conditions in both phases are almost the same, but there is more stability at gas phase rather than water phase. Likewise to comparing the electrostatic energy between two phases, the most stability of water phase is $-28398.7 \mathrm{Kcal} / \mathrm{mol}$ value, but the most stability was seen at gas phase. It was found that maximum Vander Waals energy is $-2469.58 \mathrm{Kcal} / \mathrm{mol}$ at water phase and $\mathrm{MM}+$ force field to compare with gas phase. Also there are most gradient and bond energy at water and gas phase respectively.

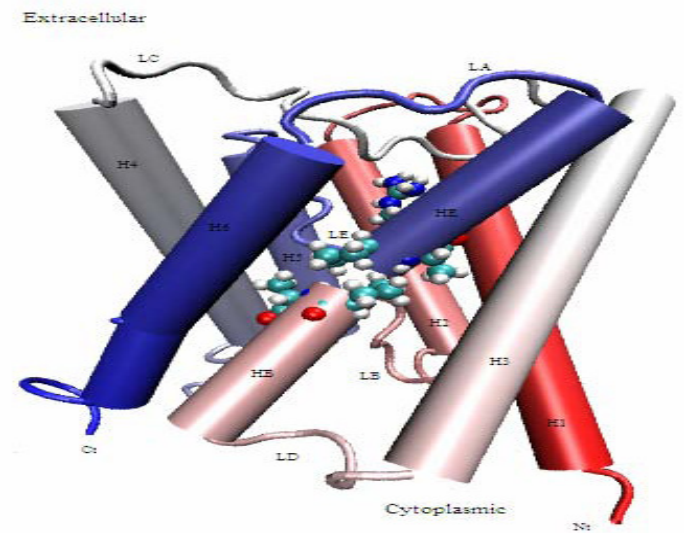

The other prominent calculated thermodynamics parameter is single point. Its bourgeon is to detect optimum point of simulation, boundary and key points. If they are demolished, molecules or proteins will lose their structures. Pursuant to Table 2, the quantities of gradient and bond energy are the most stability to 8572614.5 $\mathrm{kcal} / \mathrm{mol}$ and $190.312 \mathrm{kcal} / \mathrm{mol}$ value at water phase and gas phase respectively. The least amount of total energy at gas phase is $6613.07 \mathrm{Kcal} / \mathrm{mol}$ at $\mathrm{MM}+$ force field whereas this value at water phase is $26007 \mathrm{Kcal} / \mathrm{mol}$ in the same force field. This demonstrates that in water phase, central and vital points of this molecule are extremely unstable and

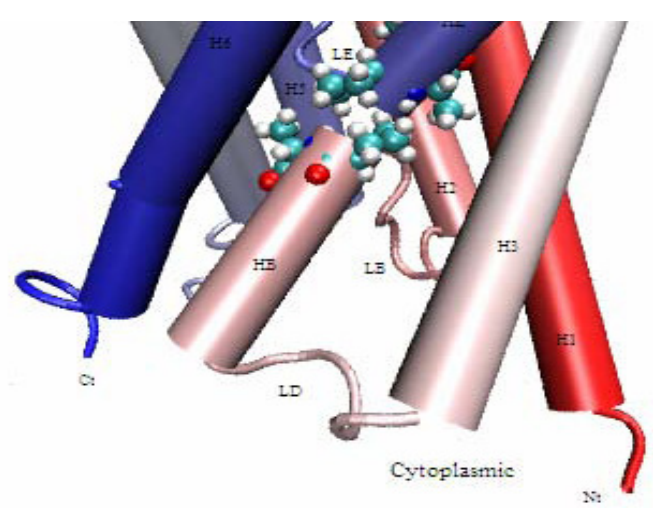

Fig 1: Normal configuration of AQP4 after minimization with CHARMM. Schematic representation of the structure of an AQP4 monomer. Side view (a) and top view from the extracellular side (b)

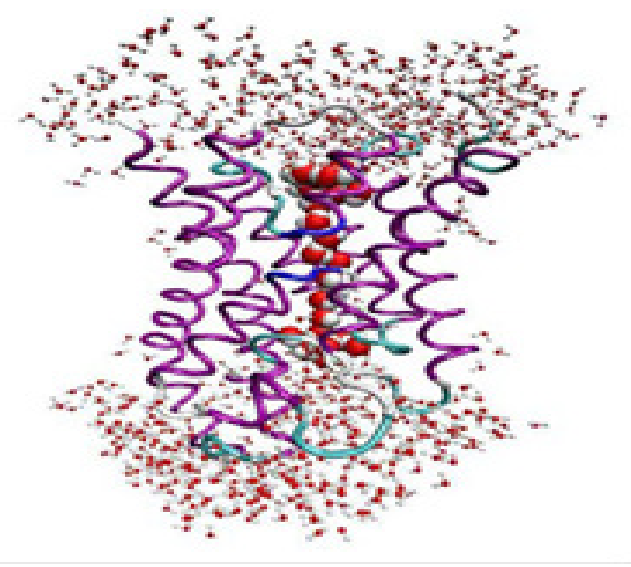

Fig. 2: Water transmission through AQP4 is accompanied by rotation of water molecule at the center of the channel.This picture was created by VMD software shows its activity, that is, in a case that molecule is active and there is no fixed point and this means survival. Moreover this molecule achieves the most of energy and there isn't any key and atom key and all of the points of molecules are apply. According to the table, the superlative electrostatic energy was seen at water phase with $-13562.6 \mathrm{Kcal} / \mathrm{mol}$ value at charm force field. Furthermore the most stability of dihedral energy and angle are in water phase at $\mathrm{MM}+$ force field and in gas phase at CHARMM phase respectively. The amount of AQP4 Vander Waals energy is the most stability at gas phase to -1134.5 $\mathrm{kcal} / \mathrm{mol}$ value.

We proceed our computation to gain the most stability of AQP4 for three basic sets of energy; Ekin, Epotential and Etotal. From a statistical point of view, the obtained valuable data of above mentioned thermodynamic parameters, analyzed under different 
simulation procedure and force fields at 100 ps span for molecular dynamics and monte carlo, and 1 ps for langevin are listed in 3,4 and 5 Tables.

Table 1 is depicts AQP4 kinetic energy at three various force fields and molecular dynamics and langevin methods.

Molecular dynamics is simulation method especially for macromolecules such as proteins. It is based on Newton's equations of motion and it is an important tool to realize the structure and function of biological macromolecules. The Langevin Dynamics simulation is an important method which is also applied for structure and function of the molecule. This is as the same as molecular dynamics, but it is a quick method and is the best of molecular dynamics when used in salvation box (in water box).

According to the results, the most amounts of kinetin energy and acceleration was seen in gas phase and charm force field to $2820 \mathrm{kcal} / \mathrm{mol}$ value.

We know that the optimum functions of biological systems are arisen at low energy levels. Therefore the maximum amount of favorable potential energy of AQP4 is seen at water phase and amber force field to $-1160.06 \mathrm{kcal} / \mathrm{mol}$ value. Analysis of data on table 3 shows that optimum total energy of AQP4 and high stability occurs at langevin method, water phase and MM+ force field.

\section{CONCLUSION}

Aquaporin-4 (AQP4) is an osmoreceptor that forms a water-specific channel to regulate body water balance and to mediate water flow within the central nervous system.

The starting point of the present study is profound to manifest thermodynamics characteristics of impressive AQP4 role in biology. In order to clarify the majestic AQP4 role in biology, it was modeled. The simulation assay accomplished by applying the HYPERCHEM software related to various force fields and basis sets of chemical computational methods as mentioned in the context. The increase in computational speed occurs at QM/ $\mathrm{MM}$ implementation trajectory that enforces each other, and gives us interesting insights into energetic attributes of AQP4 molecule. As a consequence, according to obtained data we inferred that there was an increase in the relative Etot of the AQP4 and the extremities quantity to convey water at water phase. Likewise the study highlighted that the lowest amount of electrostatic, Vander Waals, potential and total energy and therefore the fine stability of AQP4 at water phase, means the condition there is a condition in living cell membrane.

\section{REFERENCES}

1. MacAulay, N.,Hamann, S.and Zeuthen, T., Water transport in the brain: role of cotransports. Neuroscience.2004; 129: 10311044.

2. Amiry-Moghaddam,M and Ottersen, O.P., The molecular basis of water transport in the brain. Nat Rev Neurosci. 2003; 4: 991-1001.

3. Simard,M. and Nedergaard, M., The neurobiology of glia in the context of water and ion homeostasis. Neuroscience. 2004; 129: 877-896.

4. Preston,G.M., Carroll, T.P.,Guggino, W.B. and Agre, P., Appearanceof water channels in Xenopus oocytes expressing red cell CHIP28 protein.Science. 1992; 256: 385-387.

5. King, L.S., Kozono., D. and Agre , P., From structure to disease: the evolving tale of aquaporin biology. Nat Rev Mol Cell Biol. 2004; 5: 687-698.

6. Agre, P., King, L.S., Yasui, M., Guggino, W.B., Ottersen,O.P., Fujiyoshi, Y., Engel, A., and Nielsen, S., Aquaporin water channels-from atomic structure to clinical medicine.J Physiol. 2002; 542: 3-16.

7. Masanori, H.,Akinori, K., Mitsunori, k.,Water transport in Aquaporins: Osmotic Permeability Matrix Analysis of Molecular Dynamics Simulations, Biophysical Journal, 2007; 93(2): 373-385

8. Walter L. Ash1, Marian R. Zlomislic, Eliud O. Oloo1, D. Peter Tieleman, Computer simulations of membrane proteins, Department of Biological Sciences, University of Calgary, 2500 University Dr. NW, 
9. Yubao, C., Bastien, D.A., Water transport in human aquaporin-4: Molecular dynamics (MD) simulations, Biochemical and Biophysical Research Communications Journal, 2011; 412(4): 654-659.

10. Borgnia, M., S. Nielsen, A. Engel, and P. Agre, Cellular and molecular biology of the aquaporin water channels. Annu. Rev. Biochem. 1999; 68: 425-458.

11. Zhu, F., E. Tajkhorshid, and K. Schulten., Collective diffusion model for water permeation through microscopic channels. Phys. Rev.Lett. 2004; 93: 224501.

12. Zhu, F., E. Tajkhorshid, and K. Schulten., Molecular dynamics study of aquaporin-1 water channel in a lipid bilayer. FEBS Lett. 2001; 504: 212-218.

13. Jensen, M. Ø., E. Tajkhorshid, and K. Schulten., The mechanism of glycerol conduction in aquaglyceroporins. Structure. 2001; 9: 1083-1093.

14. Yu, J., A. J. Yool, K. Schulten, and E. Tajkhorshid., Mechanism of gating and ion conductivity of a possible tetrameric pore in aquaporin-1.Structure. 2006; 14: 1411-1423.

15. Borgnia, M. J., D. Kozono, G. Calamita, P. C. Maloney, and P. Agre., Functional reconstitution and characterization of AqpZ, theE. coli water channel protein. J. Mol. Biol. 1999; 291: 1169-1179.

16. Mackerell, A. D., Jr., Empirical force fields for biological macromolecules: overview and issues. J. Comput. Chem. 2004; 25: 15841604.

17. Feller, S. E., and A. D. MacKerell, Jr., An improved empirical potential energy function for molecular simulations of phospholipids. $J$. Phys. Chem. B. 2000; 104: 7510-7515.

18. Mackerell, A. D., Jr., M. Feig, and C. L. Brooks III., Extending the treatment of backbone energetics in protein force fields: limitations of gas-phase quantum mechanics in reproducing protein conformational distributions in molecular dynamics simulations. J. Comput. Chem. 2004; 25: 1400-1415.

19. MacKerell, A. D., Jr., M. Feig, and C. L. Brooks III., Improved treatment of the protein backbone in empirical force fields. J. Am. Chem. Soc. 2004; 126: 698-699.

20. Peter Agre, David Kozono, Aquaporin water channels: molecular mechanisms for human diseases, Departments of Biological Chemistry and Medicine, Johns Hopkins School of Medicine, Baltimore, MD 212052185, USA

21. F. Kaveh, K. zare1, S. Salemi1, A. R. Ilkhani and N. Khodayari., Molecular Dynamics Simulation of Water Transportation through Aquaporin-4 in Rat Brain Cells, J.Phys. Theor. Chem.IAU Iran: Winter 2009; 5(4): 177-18

22. Noam Bernstein, ${ }^{*} A$ Csilla Várnai, B Iván Solt, C Steven A. Winfield, D Mike C. Payne, D István Simon,c Mónika Fuxreiterc and Gábor Csányib. QM/MM simulation of liquid water with an adaptive quantum region. Phys. Chem. Chem. Phys., 2012; 14: 646-656

23. MacAulay, N \& Zeuthen, T., 'Water transport between CNS compartments: contributions of aquaporins and cotransporters' Neuroscience, 2010; 168(4): 941-56. 